\title{
Dimercaptosuccinic acid in combination with carbapenems against isogenic strains of Escherichia coli producing or not producing a metallo- $\beta$-lactamase in vitro and in murine peritonitis
}

\author{
G. Cheminet ${ }^{1}$, V. de Lastours ${ }^{1,2}$, L. Poirel ${ }^{3,4}$, F. Chau $^{1}$, K. Peoc'h ${ }^{5,6}$, L. Massias ${ }^{1,7}$, B. Fantin (D) ${ }^{1,2 *} \dagger$ and \\ P. Nordmann ${ }^{3,4} \dagger$
}

\begin{abstract}
${ }^{1}$ Université de Paris, IAME, UMR 1137 INSERM, F-75018 Paris, France; ${ }^{2}$ Médecine interne, Hôpital Beaujon, AP-HP Nord, Université de Paris, F-92110 Clichy, France; ${ }^{3}$ IAME, UMR 1137 Laboratoire Européen Associé INSERM, Université de Fribourg, Fribourg, Switzerland;

${ }^{4}$ Microbiologie Médicale et Moléculaire, Département de Médecine, Faculté des Sciences et de Médecine, Centre de Référence des Résistances Emergentes aux Antibiotiques (NARA), Université de Fribourg, Fribourg, Switzerland; ${ }^{5}$ Université de Paris, CRI, UMR 1149 INSERM, F-75018 Paris, France; ${ }^{6}$ Laboratoire de biochimie, Hôpital Beaujon, AP-HP Nord, F-92110 Clichy, France; ${ }^{7}$ Laboratoire de pharmacologie et toxicologie, AP-HP Nord, Hôpital Bichat-Claude Bernard, F-75018 Paris, France
\end{abstract}

${ }^{*}$ Corresponding author. E-mail: bruno.fantin@aphp.fr †Joint last authors.

\begin{abstract}
Background: Carbapenemase-producing Enterobacterales represent a major therapeutic challenge. MBLs, requiring zinc at their catalytic site, could be inhibited by meso-dimercaptosuccinic acid (DMSA), a heavy metal chelator already widely used for treating lead intoxication.
\end{abstract}

Objectives: To evaluate the activity of carbapenems alone or combined with DMSA against MBL-producing Escherichia coli in a severe murine peritonitis model.

Methods: Isogenic strains of wild-type E. coli CFT073 producing the MBLs NDM-1, VIM-2 and IMP-1, and the control serine carbapenemases OXA-48 and KPC-3 were constructed. MIC determinations and time-kill assays were performed for imipenem, meropenem and ertapenem alone or in combination with DMSA. Infected mice were treated intraperitoneally for $24 \mathrm{~h}$ with imipenem, DMSA or their combination. Bacterial counts in peritoneal fluid and spleen were assessed at $24 \mathrm{~h}$.

Results: DMSA in combination with each carbapenem caused a significant decrease in the MICs for all MBLproducing strains, in a concentration-dependent manner, but did not provide benefit against non-MBL strains. In mice infected with the NDM-1-producing strain, the combination of imipenem and DMSA significantly reduced bacterial counts in peritoneal fluid $(P=0.0006)$ and spleen $(P<0.0001)$, as compared with imipenem alone, with no benefit against the KPC-3-producing and CFT073 strains. DMSA concentrations in plasma of mice were comparable to those obtained in humans with a standard oral dose.

Conclusions: DMSA restores the activity of carbapenems against MBL-producing strains, and its combination with carbapenems appears to be a promising strategy for the treatment of NDM-producing E. coli infections.

\section{Introduction}

Antimicrobial resistance is a significant public health concern, leading to millions of deaths and high costs across the globe. ${ }^{1,2}$ Notably, the spread of ESBL-producing Enterobacterales, resistant to third-generation cephalosporins and causing both communityacquired and healthcare-associated infections, has led to an increase in consumption of carbapenems., ${ }^{3,4}$ More recently, carbapenem-resistant Enterobacterales have emerged worldwide, especially carbapenemase-producing Enterobacterales
(CPE), which represent a major therapeutic challenge. ${ }^{5}$ CPE often carry plasmids encoding resistance to carbapenems as well as to quinolones, aminoglycosides or co-trimoxazole, ${ }^{6}$ leaving few therapeutic options and leading to high mortality rates for CPE infections. ${ }^{7,8}$ These MDR-carrying plasmids are now found in Escherichia coli, which is both a major commensal and the most frequent pathogen responsible for urinary tract and intestinal infections. ${ }^{9}$ Thus, finding alternative therapeutic options against CPE is an urgent medical need. 
Among carbapenemases, New Delhi metallo- $\beta$-lactamase 1 (NDM-1) is of particular concern because of its rapid spread, particularly in E. coli, and the limited therapeutic options. ${ }^{10}$ Indeed, NDM-1 is an MBL that hydrolyses all $\beta$-lactams except aztreonam, ${ }^{11}$ including the newly developed combinations of a cephalosporin and $\beta$-lactamase inhibitor. Those novel combinations such as ceftazidime/avibactam are effective only against some types of CPE, i.e. serine carbapenemase producers (OXA-48/KPC). ${ }^{8,12,13}$ Even though several in vitro studies have described different strategies to inhibit the activity of NDM-1, no specific clinical inhibitor is available to date.

In order to hydrolyse $\beta$-lactams, MBLs require zinc ions at their active site. Meso-dimercaptosuccinic acid (DMSA; generic name: succimer) is an orally administered heavy metal chelator approved for years in many countries for the treatment of lead and mercury intoxication. ${ }^{14,15}$ It is safe in children and adults, ${ }^{15,16}$ with a large therapeutic window, as it is less toxic than other chelators that have been used therapeutically. ${ }^{17}$ We hypothesized that DMSA might have a similar chelating activity on the zinc-carbapenemase binding, allowing, when combined with a carbapenem, restoration of the activity of the carbapenem against MBL producers.

This work aimed to evaluate the activity of carbapenems alone or in combination with DMSA against MBL-producing E. coli both in vitro and in a severe murine peritonitis model.

\section{(1)}

\section{Materials and methods}

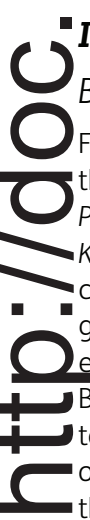
In vitro studies

Bacterial strains, plasmids and growth conditions

Five clinical isolates were used as the source of carbapenemases genes for the construction of isogenic strains: Klebsiella pneumoniae NDM-1 (R2726), Pseudomonas aeruginosa VIM-2 (R51), P. aeruginosa IMP-1 (R409), K. pneumoniae OXA-48 (11978) and K. pneumoniae KPC-3 (GF). Chemically competent E. coli TOP10 was used for cloning experiments, and uropathogenic E. coli CFT073 (O6: K2: H1), previously used in murine peritonitis models, served as the recipient strain. ${ }^{18,19}$ Plasmid pACYC184 (New England Biolabs, Ipswich, MA, USA), which carries chloramphenicol (cat) and tetracycline (tet) resistance genes, was used for cloning experiments. In order to ensure plasmid maintenance, all subcultures were performed in the presence of ampicillin $100 \mathrm{mg} / \mathrm{L}$ and chloramphenicol $20 \mathrm{mg} / \mathrm{L}$. Regions corresponding to structural genes and promoters of bla $a_{\mathrm{NDM}-1}, b / a_{\mathrm{VIM}-2}$, bla $a_{\mathrm{IMP}-1}$, bla $_{\mathrm{OXA}-48}$ and bla $a_{\mathrm{KPC}-3}$ were amplified by PCR and cloned into pACYC184 (primers used are described in Table S1, available as Supplementary data at JAC Online). E. coli CFT073 was transformed with recombinant plasmids by electroporation, resulting in CFT073pACYC184-NDM-1, CFT073-pACYC184-VIM-2, CFT073-pACYC184-IMP-1, CFT073-pACYC184-OXA-48, CFT073-pACYC184-KPC-3 and CFT073pACYC184 (non-recombinant and cloning plasmid). They will be referred to as CFT073-NDM-1, CFT073-VIM-2, CFT073-IMP-1, CFT073-OXA-48, CFT073-KPC-3 and CFT073, respectively.

\section{Minimum inhibitory concentrations}

MICs of imipenem (Imipenem Mylan ${ }^{\circledR}$, Saint Priest, France), meropenem (Meropenem Kabi ${ }^{\circledR}$, Sèvres, France) and ertapenem (Invanz ${ }^{\circledR}$, Merck, Haarlem, the Netherlands) alone or in the presence of DMSA powder (Sigma-Aldrich, Saint-Quentin-Fallavier, France) at different concentrations $(0.3,1.5,3,6,9$ and $12 \mathrm{mM})$, were determined by the broth microdilution method, according to EUCAST (http://www.eucast.org/), in triplicate.

\section{Bactericidal combination study}

The presence of an in vitro synergy between imipenem and DMSA was tested by performing time-kill assays against CFT073, CFT073-NDM-1, CFT073-OXA-48 and CFT073-KPC-3 with imipenem at a concentration equal to the MIC against the studied strain and DMSA at $6 \mathrm{mM}$, alone or in combination, as previously described. ${ }^{20}$ Standard Mueller-Hinton (MH) broth test tubes were used as controls. A bactericidal effect was defined as a decrease of $\geq 3 \log _{10}$ in cfu counts after $24 \mathrm{~h}$ compared with the initial inoculum. A synergistic effect was defined as $a \geq 2 \log _{10}$ decrease in cfu counts after $24 \mathrm{~h}$ between the combination and its most active constituent. ${ }^{20}$ All in vitro experiments were repeated at least three times, and the median values are reported.

\section{Bacterial fitness}

Bacterial fitness was determined by measuring growth rates at $37^{\circ} \mathrm{C}$ in $\mathrm{MH}$ broth as described. ${ }^{21}$ For each strain and condition, maximal growth rate and time to achieve maximal growth rate were measured in three independent experiments and the median values were reported for each strain.

\section{Murine peritoneal infection model}

Ethics

Animal experiments and laboratory protocols were approved by the Departmental Direction of Veterinary Services (agreement No. 75-861). The peritonitis protocol was approved by the French Ministry of Research and by the ethics committee for animal experiments (No. APAFIS 49492016021215347422).

\section{Peritonitis model}

We used the lethal murine intra-abdominal infection model previously developed by our group. ${ }^{18,19}$ Five-week-old Swiss female mice weighing 25-30 g were inoculated by the IP route with $250 \mu \mathrm{L}$ of a bacterial suspension in porcine mucin $10 \%$ (Sigma-Aldrich), corresponding to a final inoculum of approximately $2.5 \times 10^{7} \mathrm{cfu} / \mathrm{mL}$. The strains tested were CFT073, CFT073-NDM-1 and CFT073-KPC-3. Two hours after inoculation, at least five mice per strain were sacrificed to determine the pre-therapeutic bacterial loads, and referred to as start-of-treatment controls, since all untreated mice died within $24 \mathrm{~h}$, preventing the use of end-of-treatment controls. For the treated mice, IP injections of the tested compounds were started $2 \mathrm{~h}$ after inoculation and continued for $24 \mathrm{~h}$ (identical preliminary results were obtained using the SC route of administration of antibiotics). Mice were sacrificed by IP injection of $200 \mu \mathrm{L}$ of pentobarbital $40 \%$ (Euthasol Vet ${ }^{\circledR}$, Dechra Veterinary Products, France) $4 \mathrm{~h}$ after the last antibiotic injection. Immediately after death, a peritoneal wash was performed by IP injection of $2 \mathrm{~mL}$ of sterile saline solution, followed by a gentle massage of the abdomen and opening of the peritoneum to collect $1 \mathrm{~mL}$ of peritoneal fluid. The spleen was extracted and homogenized. Samples were plated on LB agar supplemented with chloramphenicol $20 \mathrm{mg} / \mathrm{L}$ and ampicillin $100 \mathrm{mg} / \mathrm{L}$ to ensure plasmid maintenance. Colony counts were then determined after $24-48 \mathrm{~h}$ of culture. Results were expressed as $\log _{10} \mathrm{cfu} / \mathrm{g}$ of spleen and $\log _{10} \mathrm{cfu} / \mathrm{mL}$ of peritoneal fluid. The limit of detection was $1 \log _{10} \mathrm{cfu} / \mathrm{g}$ or $\mathrm{mL}$.

\section{Therapeutic regimens}

An imipenem dosing regimen of $100 \mathrm{mg} / \mathrm{kg}$ q4h was selected in order to reach the same peak plasma levels and the same percentage of time during which free drug plasma levels exceeded the MIC $\left(f T_{>M I C}\right)$ as obtained in humans against a susceptible strain with standard IV regimens. ${ }^{22,23}$ The DMSA dosing regimen was determined according to a previous study that found the $\mathrm{LD}_{50}$ of DMSA to be approximately $2500 \mathrm{mg} / \mathrm{kg}$ IP. ${ }^{24}$ We first determined the two highest dosages of DMSA that could be used in 
uninfected mice: $400 \mathrm{mg} / \mathrm{kg}$ q4h IP (2400 mg/kg per $24 \mathrm{~h}$ ) close to the previously reported $L D_{50}$ and $200 \mathrm{mg} / \mathrm{kg}$ q4h IP (1200 mg/kg per $\left.24 \mathrm{~h}\right)$. As no death or any abnormal symptom occurred for either dosing regimen after $24 \mathrm{~h}$ of treatment, the $200 \mathrm{mg} / \mathrm{kg}$ q $4 \mathrm{~h}$ regimen was selected for safety reasons as the highest DMSA dosing regimen for therapeutic experiments. First, three different therapeutic groups were evaluated in mice infected with each of the three study strains: imipenem $100 \mathrm{mg} / \mathrm{kg}$ q4h alone, DMSA $200 \mathrm{mg} / \mathrm{kg}$ q $4 \mathrm{~h}$ alone and the combination of imipenem $100 \mathrm{mg} / \mathrm{kg}$ q $4 \mathrm{~h}+$ DMSA $200 \mathrm{mg} / \mathrm{kg}$ q4h. For imipenem-based regimens, at least 10 mice per group were used ( 5 for DMSA alone). Then, the lowest effective dosing regimen of DMSA in combination with imipenem against CFT073-NDM-1 was determined: $100 \mathrm{mg} / \mathrm{kg}, 50 \mathrm{mg} / \mathrm{kg}$ and $10 \mathrm{mg} / \mathrm{kg}$ q4h (10 mice minimum per group).

\section{Assessment criteria}

Assessment criteria for each strain and each treatment regimen were: (i) the bacterial load in peritoneal fluid and spleen; (ii) the mortality rate due to infection; (iii) the percentage of sterilization of peritoneal fluid and spleen samples; and (iv) the selection of resistant mutants.

\section{Drug concentrations}

\section{mipenem/cilastatin}

After a single IP injection of imipenem/cilastatin $100 \mathrm{mg} / \mathrm{kg}$, four mice per time measurement ( $15 \mathrm{~min}, 30 \mathrm{~min}, 1 \mathrm{~h}, 3 \mathrm{~h}, 4 \mathrm{~h}$ and $6 \mathrm{~h}$ ) were anaesthetized and blood samples were obtained by intracardiac puncture before sacrifice. In addition, peritoneal washes were obtained from infected and treated mice $(n=4) 4 \mathrm{~h}$ after the last imipenem/cilastatin injection. Imipenem concentrations were measured in plasma by HPLC coupled with spectrophotometric detection at $237 \mathrm{~nm}^{25}$

\section{DMSA}

Blood samples of healthy mice were collected by intracardiac puncture 30 and $60 \mathrm{~min}$ after a single IP dose of DMSA $400 \mathrm{mg} / \mathrm{kg}, 200 \mathrm{mg} / \mathrm{kg}$, $100 \mathrm{mg} / \mathrm{kg}, 50 \mathrm{mg} / \mathrm{kg}$ and $10 \mathrm{mg} / \mathrm{kg}$, and stored at $-80^{\circ} \mathrm{C}$.

An analytical technique of dosage of DMSA was developed. For each house, $80 \mu \mathrm{L}$ of plasma was sampled into a $1.5 \mathrm{~mL}$ microtube and mixed with $20 \mu \mathrm{L}$ of $10 \%$ trichloroacetic acid (TCA) to precipitate the proteins and extract the component. Samples were vortexed for $5 \mathrm{~min}$, then placed in an ultrasound bath for $1 \mathrm{~min}$ and centrifuged to sediment the proteins (15 $000 \mathrm{~g}, 5 \mathrm{~min}$ at $16^{\circ} \mathrm{C}$ ). Supernatants were analysed using MS coupled with a triple quadripole Shimadzu LC-MS 8030 (LC-MS/MS).

The separation was performed on a kinetex polar column $2.6 \mu \mathrm{m} \mathrm{C18}$ $100 \mathrm{~A} 50 \times 2.1 \mathrm{~mm}$ (Phenomenex) at $40^{\circ} \mathrm{C}$ with an injection volume of $10 \mu \mathrm{L}$. The mobile phase consisted of a mix of A ( $0.5 \%$ formic acid in water) and $\mathrm{B}$ (acetonitrile). Initial conditions were $99 \% \mathrm{~A}$ and $1 \% \mathrm{~B}$. After $0.5 \mathrm{~min}$, the gradient decreased until $1.2 \mathrm{~min}$ to $5 \% \mathrm{~A}$ and $95 \% \mathrm{~B}$ and remained as such for $0.2 \mathrm{~min}$. It then rapidly came back to the initial composition between $1.42 \mathrm{~min}$ and $2.8 \mathrm{~min}$. The mobile phase flow was $0.5 \mathrm{~mL} / \mathrm{min}$.

DMSA is an unstable molecule in plasma. All the compound was degraded after a few minutes as it oxidizes completely into a dimer of two molecules linked by a di-sulfur bridge. We therefore quantified the degradation compound rather than DMSA. The multiple reaction monitoring (MRM) analysis on precursor and fragmented ions of DMSA was chosen for quantification. The analysis was performed by negative ionization, and the precursor ion of the oxidized compound corresponded to $360.90 \mathrm{~m} / \mathrm{z}$ and the chosen fragmented ions to $102.90 \mathrm{~m} / \mathrm{z}$ and $146.90 \mathrm{~m} / \mathrm{z}$. For DMSA, they were, respectively, $181.1,103.0$ and $147.0 \mathrm{~m} / \mathrm{z}$. The limit of detection was $0.05 \mu \mathrm{M}$ and the mean variation for each sample determination was $9 \%$.
Zinc

Zinc concentrations were measured in $\mathrm{MH}$ broth and mouse sera, by inductively coupled plasma MS (ICP-MS) on an X-Series $\mathrm{II}^{\circledR}$ from Thermo Scientific. ${ }^{26}$

\section{Statistical analyses}

Continuous variables were expressed as the median and ranges (minimum to maximum) and compared using non-parametric test (Kruskall-Wallis). Proportions were compared using the Fisher exact test. Statistical analyses were performed with $R^{\circledR}$ software (version 3.6.0). A $P$ value $\leq 0.05$ was considered significant.

\section{Results}

\section{In vitro experiments}

\section{MICs of carbapenems alone or in combination with DMSA}

The MICs of imipenem, meropenem and ertapenem alone or in combination with increasing concentrations of DMSA against study strains (susceptible strain E. coli CFT073 and isogenic derivatives producing NDM-1, VIM-2, IMP-1, OXA-48 or KPC-3) are shown in Table S2. DMSA supplementation generated a significant reduction in carbapenem MICs in a concentration-dependent manner and a full recovery of susceptibility to carbapenems for MBL-producing strains at a maximum concentration of $6 \mathrm{mM}(\sim 1.1 \mathrm{~g} / \mathrm{L})$. The concentration of DMSA that allowed each carbapenem to reach its susceptibility breakpoint varied depending on the carbapenemase and the carbapenem, from $6 \mathrm{mM}$ for NDM-1-, 1.5-3 mM for VIM-2and $0.3-1.5 \mathrm{mM}$ for IMP-1-producing strains. No significant effect was observed against non-MBL-producing strains or the WT strain. The median concentration of zinc in $\mathrm{MH}$ broth was $1.1 \mathrm{mg} / \mathrm{L}$ (0.02 mM) (range 1.09-1.18 mg/L).

\section{Time-kill assays}

The results of time-kill curves for the three strains studied in vivo are represented in Figure 1. A beneficial bactericidal effect between imipenem at the MIC and DMSA $6 \mathrm{mM}$ was observed against CFT073-NDM-1, with a $>3 \log _{10} \mathrm{cfu} / \mathrm{mL}$ reduction in bacterial load at $24 \mathrm{~h}$, but not against the susceptible or non-MBL-producing resistant strains.

\section{Bacterial fitness}

No difference of the maximal growth rate or time to achieve maximal growth rate was observed for each strain in MH broth containing DMSA or not at concentrations up to $6 \mathrm{mM}$ (data not shown).

\section{Murine peritoneal infection model}

\section{Activity of imipenem and DMSA alone or in combination}

DMSA alone at the highest dose tested $(200 \mathrm{mg} / \mathrm{kg} \mathrm{q} 4 \mathrm{~h}$ ) had no antibacterial effect in mice against any of the study strains (Figure 2). Since the mortality in DMSA-alone treatment groups was $100 \%$, no peritoneal fluid samples were available. In contrast, imipenem alone at a dose of $100 \mathrm{mg} / \mathrm{kg}$ q4h showed a significant antibacterial activity in peritoneal fluid and spleen as compared with controls against the susceptible strain and against the NDM-1 strain, but no significant effect in spleen against the KPC-3 strain 

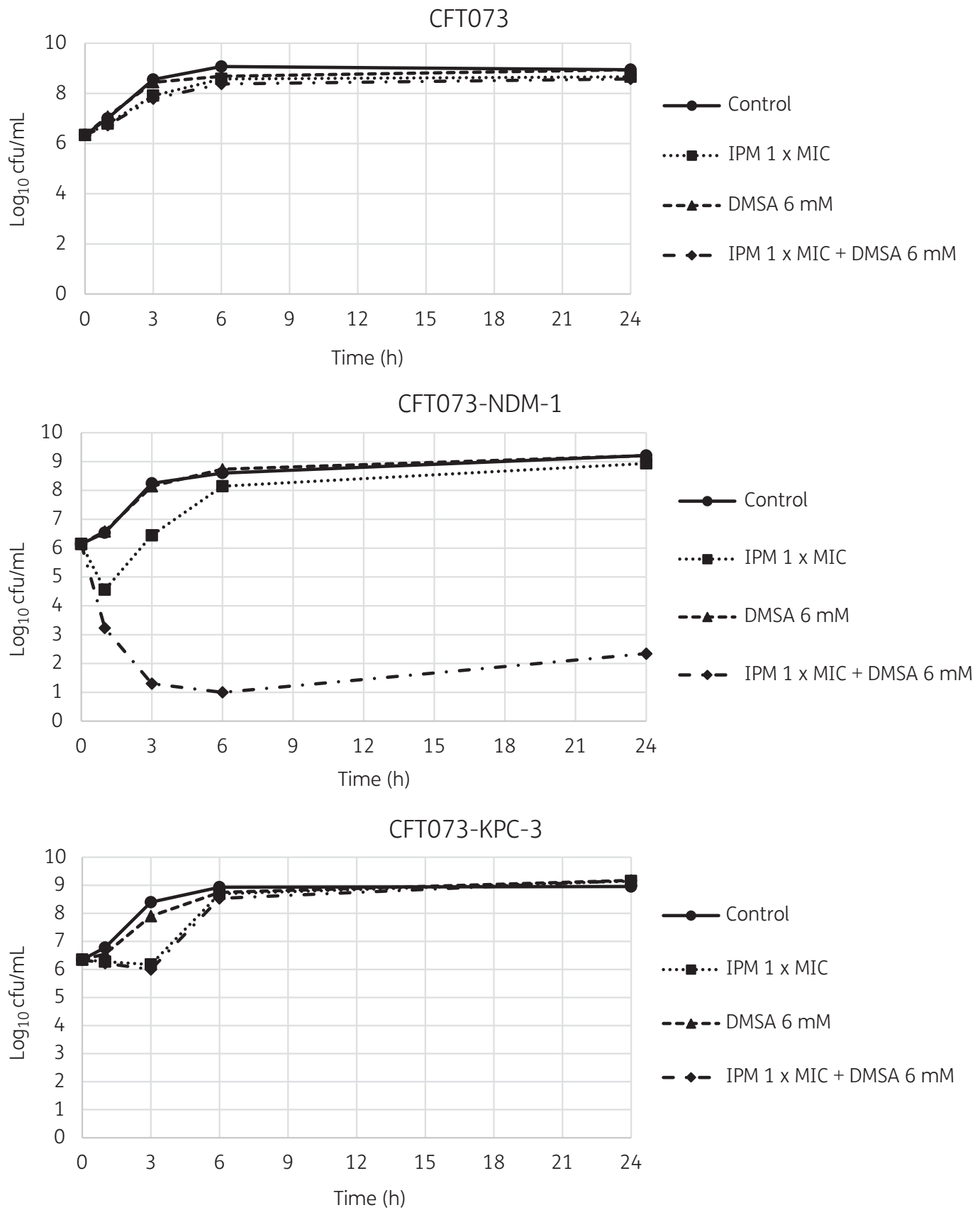

Figure 1. Time-kill curves of imipenem (IPM) and DMSA alone or in combination against WT CFT073 (top), CFT073- NDM-1 (middle) and CFT073-KPC3 (bottom). Imipenem MIC was respectively, 0.125, 64 and 8 mg/L for CFT073, CFT073-NDM-1 and CFT073-KPC-3.

(Figure 2). In CFT073-NDM-1-infected mice, the combination of imipenem and DMSA produced a significant reduction of bacterial load in peritoneal fluid and spleen at $24 \mathrm{~h}$ as compared with imipenem alone $\left(-1.35 \log _{10} \mathrm{cfu} / \mathrm{mL}\right.$ in peritoneal fluid, $P=0.0006$ and $-1.04 \log _{10} \mathrm{cfu} / \mathrm{g}$ in spleen, $\left.P<0.0001\right)$. No significant benefit of the combination was achieved in mice infected with the nonMBL-producing strains CFT073 and CFT073-KPC-3 as compared with imipenem alone. The dose-response study performed in CFT073-NDM-1-infected mice with decreasing DMSA dosing regimens of 100,50 and $10 \mathrm{mg} / \mathrm{kg}$ q4h showed that a significant reduction in bacterial counts was still achieved with the $50 \mathrm{mg} / \mathrm{kg}$ $\mathrm{q} 4 \mathrm{~h}$ dosing regimen in the spleen in contrast to peritoneal fluid where the $200 \mathrm{mg} / \mathrm{kg}$ q $4 \mathrm{~h}$ regimen was the only tested DMSA dose found to be effective (Figure 3).

\section{Sterilization rates}

No spleen sterilization was obtained for any strain and therapeutic regimen. In peritoneal fluid, the imipenem sterilization rate was $0 \%(0 / 12)$ against NDM-1 and $12 \%(2 / 17)$ in combination with 


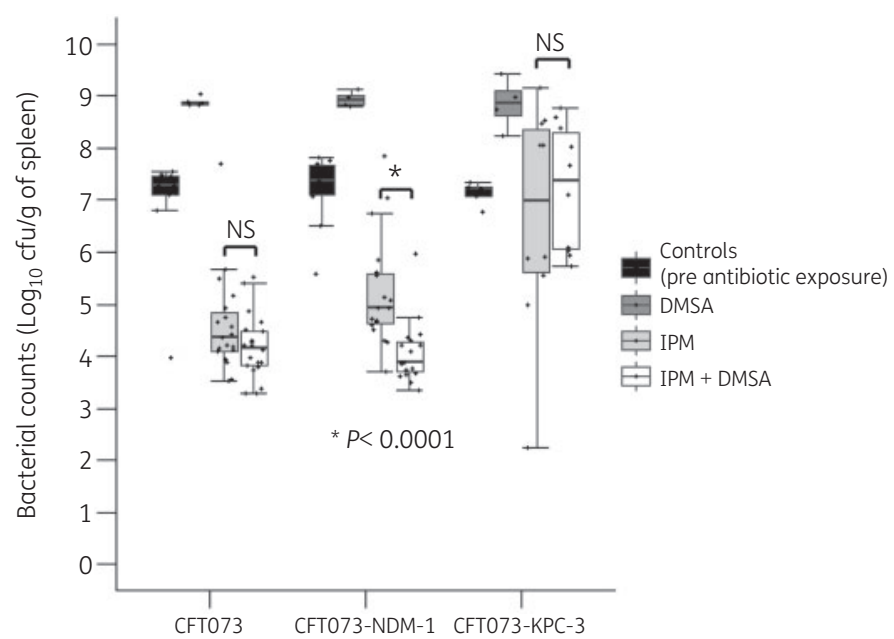

Figure 2. Bacterial counts in the spleen from mice infected with $E$. coli CFT073, CFT073-KPC-3 and CFT073-NDM-1 strains before antibiotic exposure (Controls, pre-antibiotic exposure) and after $24 \mathrm{~h}$ of treatment with DMSA alone (DMSA), imipenem alone (IPM) or in combination (IPM + DMSA). Each point corresponds to a mouse. Boxplots represent the median (horizontal black line), IQR and minimum/maximum values. Mice were infected with E. coli CFT073, CFT073-NDM-1 or CFT073-KPC-3 strains just before antibiotic exposure (Controls, $n=11,10$, and 5 , respectively) and after treatment with DMSA alone (DMSA, $n=5,4$, and 4 , respectively), imipenem alone (IPM, $n=19,19$, and 10 respectively) or in combination (IPM + DMSA, $n=20,19$, and 10 , respectively) for $24 \mathrm{~h}$. NS, no significant difference.

DMSA ( $P=0.49)$, similar to the $13 \%(2 / 15)$ obtained with imipenem alone against the susceptible strain.

\section{Mortality rates}

The mortality rates in groups treated by DMSA alone were 100\% - for all strains within $24 \mathrm{~h}$. No significant reduction of mortality was obtained with the combination of imipenem and DMSA as compared with imipenem alone against any of the three tested strains. In particular, the mortality rate for CFT073-NDM-1 was $37 \%(7 / 19)$ for imipenem alone versus $11 \%(2 / 19)$ for the combination $(P=0.13)$.

\section{Pharmacokinetics in mice}

\section{Imipenem/cilastatin pharmacokinetics}

Peak levels of imipenem/cilastatin in plasma were achieved 15 min after IP injection. The median concentration was $86 \mathrm{mg} / \mathrm{L}$ (range 10-125), corresponding to a free concentration of $57 \mathrm{mg} / \mathrm{L}$. The $\mathrm{fT}_{>\mathrm{MIC}}$ values in mice were $66 \%, 0 \%$ and $27 \%$ for strains CFT073, CFT073-NDM-1 and CFT073-KPC-3, respectively. Imipenem concentrations were below the detection limit $(0.5 \mathrm{mg} / \mathrm{L})$ $4 \mathrm{~h}$ after the end of the treatment in infected mice.

\section{DMSA pharmacokinetics}

DMSA peak levels in plasma were achieved 30 min after a single IP dose of 100, 200 or $400 \mathrm{mg} / \mathrm{kg}$ ( $n=3$ for each dose) in uninfected mice and it was not detected after $2 \mathrm{~h}$. Results are shown in
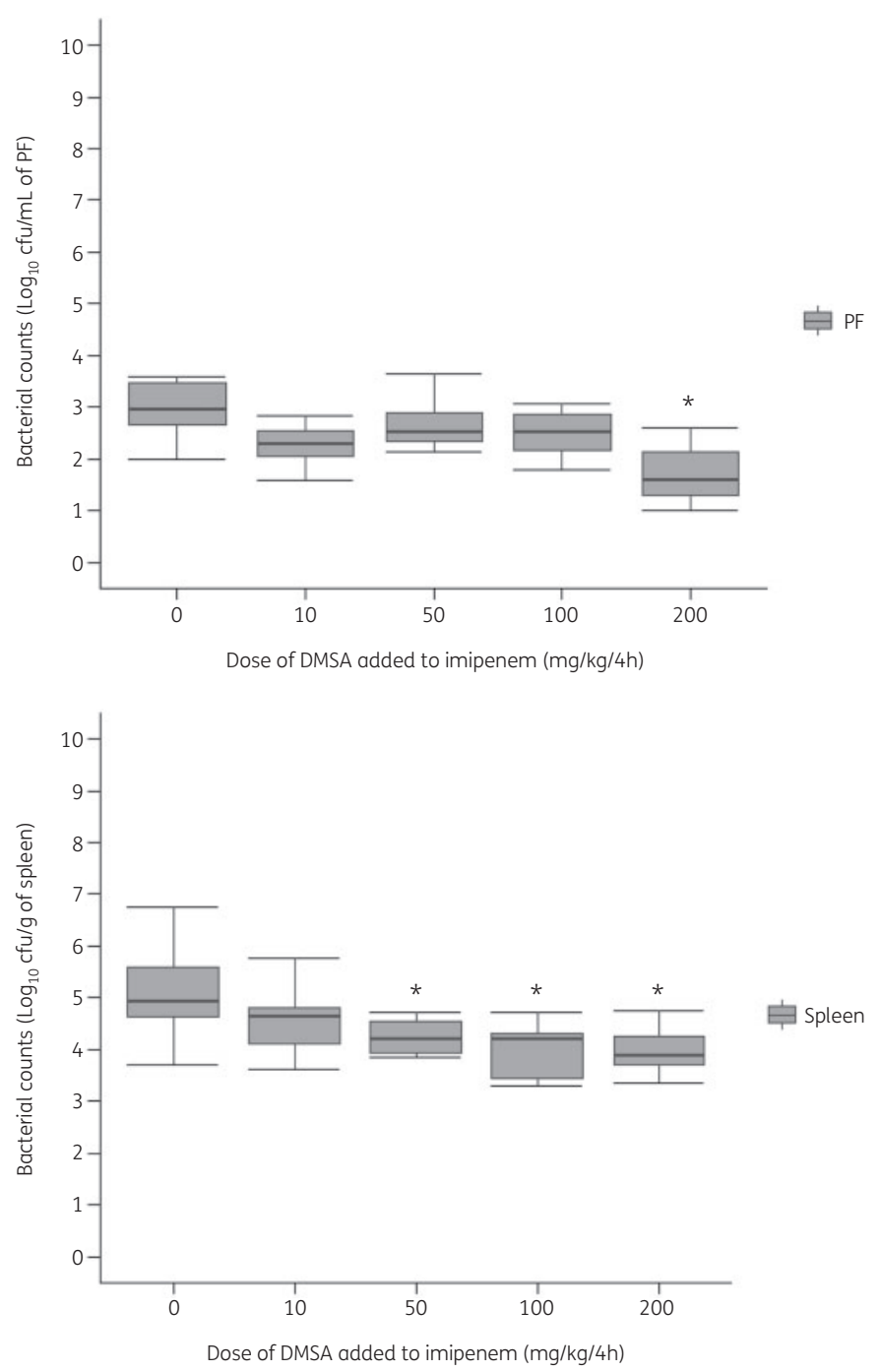

Figure 3. Bacterial counts after a $24 \mathrm{~h}$ treatment with imipenem alone or in combination with increasing doses of DMSA in the peritoneal fluid (PF; top) and in the spleen (bottom) from mice with peritonitis due to the E. coli CFT073-NDM-1 strain. Boxplots represent the median (black line), IQR and minimum/maximum values. The number of mice was 19, 10, 10, 10, 19 and 17, 10, 9, 8, 12 for DMSA 0, 10, 50, 100 and $200 \mathrm{mg} / \mathrm{kg} / 4$ $\mathrm{h}$, respectively, in spleen and PF, respectively. ${ }^{*} P<0.05$ compared with DMSA $0 \mathrm{mg} / \mathrm{kg} / 4 \mathrm{~h}$ (imipenem alone).

Table S3 and compared with human values after a single oral dose of $10 \mathrm{mg} / \mathrm{kg}$.

\section{Zinc concentrations}

The median concentration of zinc in the sera from healthy mice was $2.02 \mathrm{mg} / \mathrm{L}$ (range 1.59-3.36) and was $2.72 \mathrm{mg} / \mathrm{L}$ (range 1.94-3.87) in infected mice treated during $24 \mathrm{~h}$ with imipenem and DMSA $(P=0.41)$.

\section{Discussion}

In this study, DMSA allowed a recovery of the in vitro activity of carbapenems against MBL-producing E. coli strains, and achieved a 
significant benefit in combination with imipenem on the reduction of bacterial loads in peritoneal fluid and spleen in a lethal murine peritonitis model against an NDM-1-producing E. coli strain. This effect was not found with isogenic non-MBL-producing strains (OXA48 or KPC producers), suggesting a specific effect on MBL.

An unexpected finding was that the beneficial antibacterial effect of adding DMSA to imipenem was achieved in vivo with plasma concentrations in the micromolar range whereas in vitro concentrations necessary to achieve this effect were in the millimolar range (1000-fold higher) (Tables S2 and S3). At least two hypotheses might explain this result. First, the rapid oxidation of DMSA observed in plasma could lead to the production of a more active dimer, thus enhancing its chelator activity in vivo. Indeed, the dimer is still a chelator as the carboxylic acid groups are not modified and the di-sulfur is still available. All the functions that bind to metal ions are still present in the new molecule. Additional studies are thus required to test the specific activity of the dimer, relative to DMSA. Second, the unexpected activity of imipenem in vivo against the NDM-1 strain could be associated with a decreased requirement of DMSA concentrations to achieve a beneficial activity of the combination in mice. This unexpected activity - of imipenem in vivo corroborates results obtained by others in the thigh murine infection model without clear explanation. ${ }^{27,28}$ Such an effect could not be explained by a fitness defect of the NDM-1producing strain, nor by the IP route, as imipenem pharmacokinetics in plasma were similar to those with the SC route. ${ }^{25}$ The pharmacokinetic/pharmacodynamic profile of imipenem in mice could not be an explanation since the $\mathrm{fT}_{>\mathrm{MIC}}$ was $0 \%$ for CFT073NDM-1 herein, suggesting that mechanisms other than antimicrobial activity of supra-MIC concentrations of carbapenems could be involved. ${ }^{29}$ Differences in zinc concentrations and consequently of carbapenemase activity were excluded, since similar concentrations of zinc were measured in plasma and in MH medium.

In vitro, the combination of DMSA with imipenem, meropenem or ertapenem produced a significant decrease of their MIC values against MBL-producing strains in a concentration-dependent manner. Similar results were obtained with clinical isolates of NDM-1-E. coli (unpublished data, P. Nordmann). This effect was specific to MBL, as no effect was observed against the non-MBLcarbapenemase-producing strains. Moreover, it was not related to bacterial fitness modifications. MICs of carbapenems against each type of carbapenemase in the constructed isogenic strains were consistent with available data on clinical strains. ${ }^{27,28,30,31}$ The IMP1-producing strain seemed more susceptible to the effect of DMSA than others, suggesting a higher sensitivity of the IMP-1 enzyme to the amount of zinc in its environment. Of note, zinc concentrations in $\mathrm{MH}$ medium used for the determination of MICs were approximately $1 \mathrm{mg} / \mathrm{L}$, close to human concentrations in plasma. ${ }^{32}$

These in vitro results were mirrored by the in vivo results obtained in a severe peritonitis model using the constructed CFT073-NDM-1 strain. The combination of imipenem with DMSA produced a significant reduction in bacterial counts in peritoneal fluid and spleen as compared with imipenem alone, but had no effect against the non-MBL CFT073-KPC-3 strain or the susceptible control. The benefit observed with DMSA is likely to be related to its activity as a chelator of zinc, a metallic element that is essential for the hydrolytic activity of MBLs. ${ }^{33-35}$ Indeed, unlike serine- $\beta$-lactamases (such as KPC and OXA-48-type enzymes), MBLs need the coordinated action of one or two zinc ions and a water molecule to open the $\beta$-lactam ring. ${ }^{34,36}$ Several studies have described a potential interaction between DMSA and zinc. ${ }^{37,38}$ Further in vitro studies may be performed to study the intimate interactions between NDM-1, DMSA and zinc. We may study the hydrolysis of NDM-1 in the presence of increasing concentrations of DMSA followed by increasing concentration of zinc to see if the inhibition of hydrolysis of NDM-1 is reversible or irreversible.

Due to the current lack of data on the use of DMSA in such a murine model, the choice of the final DMSA dosing regimen of $1200 \mathrm{mg} / \mathrm{kg} /$ day was based on a $100 \%$ survival rate with no symptoms of toxicity. Therefore, the benefit of the addition of DMSA to imipenem was obtained for doses of DMSA much lower than the lethal doses reported in the literature. ${ }^{24}$ However, it must be acknowledged that the evaluation of the toxicity of DMSA was limited to $24 \mathrm{~h}$, corresponding to the duration of therapy in our study. A more prolonged exposure with repeated doses, closer to a standard antibiotic treatment duration, would be necessary before application in humans.

Pharmacokinetic data in humans concerning DMSA are scarce, but available studies indicate mean peak plasma concentrations of about $20-50 \mu \mathrm{M}$ after a standard $10 \mathrm{mg} / \mathrm{kg}$ oral dose (Table S3). ${ }^{16,39,40}$ These concentrations are comparable to those obtained in mice with $200 \mathrm{mg} / \mathrm{kg}$, the dose that demonstrated a beneficial effect in combination with imipenem in vivo. Thus, an in vivo benefit was obtained with plasma concentrations comparable to those achieved in humans with the standard oral dose of DMSA of $10 \mathrm{mg} / \mathrm{kg}$.

In conclusion, we provide here the proof of concept of the efficacy of DMSA in combination with carbapenems against MBLproducing $E$. coli in a lethal murine peritonitis model. Its development is following a repurposing strategy, which is intended to speed up the development of antibiotics ${ }^{41}$ that may fulfil an unmet clinical need.

\section{Acknowledgements}

We thank Sara Dion, Xavier Vuillemin, Nicolas Kieffer and Patrick Gizzi for their initial technical assistance. Geoffrey Cheminet thanks the Assistance Publique Hôpitaux de Paris and the Société de Pathologie Infectieuse de Langue Française for their financial support. This work was presented in part at Infectious Diseases week, Washington DC, USA, October 2019 (abstract no. 617) and at 'Réunion Interdisciplinaire de Chimiothérapie Anti-Infectieuse', Paris, France, December 2019.

\section{Funding}

G.C. obtained a personal grant from Assistance Publique-Hôpitaux de Paris (2018-10-14), from the Société de Pathologie Infectieuse de Langue Française (SPILF) and from the Fondation pour la Recherche Médicale (Equipe FRM 2016, grant number DEQ20161136698). This study was also supported by internal funding from IAME and by the University of Fribourg.

\section{Transparency declarations}

None to declare. 


\section{Supplementary data}

Tables S1 to S3 are available as Supplementary data at JAC Online.

\section{References}

1 Tangcharoensathien V, Sattayawutthipong W, Kanjanapimai S et al. Antimicrobial resistance: from global agenda to national strategic plan. Bull World Health Organ 2017; 95: 599-603.

2 Cassini A, Högberg LD, Plachouras D et al. Attributable deaths and disability-adjusted life-years caused by infections with antibiotic-resistant bacteria in the EU and the European economic area in 2015: a populationlevel modelling analysis. Lancet Infect Dis 2019; 19: 56-66.

3 Doi Y, Park YS, Rivera JI et al. Community-associated extended-spectrum $\beta$-lactamase-producing Escherichia coli infection in the United States. Clin Infect Dis 2013; 56: 641-8.

4 Parienti JJ, Lucet JC, Lefort A et al. Empirical therapies among adults hospitalized for community-acquired upper urinary tract infections: a decision-tree analysis of mortality, costs, and resistance. Am J Infect Control 2015; 43: e53.

5 Nordmann P, Naas T, Poirel L. Global spread of carbapenemase-producing Enterobacteriaceae. Emerg Infect Dis 2011; 17: 1791-8.

- 6 Poirel L, Bonnin RA, Nordmann P. Analysis of the resistome of a multidrugresistant NDM-1-producing Escherichia coli strain by high-throughput genome sequencing. Antimicrob Agents Chemother 2011; 55: 4224-9.

7 Karaiskos I, Lagou S, Pontikis K et al. The 'old' and the 'new' antibiotics for MDR Gram-negative pathogens: for whom, when, and how. Front Public Health 2019; 7: 151.

- 8 Wu W, Feng $Y$, Tang G et al. NDM metallo- $\beta$-lactamases and their bacterial producers in health care settings. Clin Microbiol Rev 2019; 32: e00115-8.

9 Kieffer N, Nordmann P, Aires-de-Sousa M et al. High prevalence of carbapenemase-producing Enterobacteriaceae among hospitalized children in Luanda, Angola. Antimicrob Agents Chemother 2016; 60: 6189-92.

10 Albiger B, Glasner C, Struelens MJ et al. European survey of carbapenemase-producing Enterobacteriaceae (EuSCAPE) working group. Carbapenemase-producing Enterobacteriaceae in Europe: assessment by national experts from 38 countries. Euro Surveill Bull 2015 May 2015; 20: pii=30062.

11 Queenan AM, Bush K. Carbapenemases: the versatile $\beta$-lactamases. Clin Microbiol Rev 2007; 20: 440-58.

12 Zhang J, Wang S, Wei Q et al. Synthesis and biological evaluation of Aspergillomarasmine A derivatives as novel NDM-1 inhibitor to overcome antibiotics resistance. Bioorg Med Chem 2017; 25: 5133-41.

13 Monogue ML, Giovagnoli S, Bissantz C et al. In vivo efficacy of meropenem with a novel non- $\beta$-lactam- $\beta$-lactamase inhibitor, nacubactam, against Gram-negative organisms exhibiting various resistance mechanisms in a murine complicated urinary tract infection model. Antimicrob Agents Chemother 2018; 62: e02596-17.

14 Thurtle N, Greig J, Cooney L et al. Description of 3,180 courses of chelation with dimercaptosuccinic acid in children $\leq 5 \mathrm{y}$ with severe lead poisoning in Zamfara, northern Nigeria: a retrospective analysis of programme data. PLOS Med 2014; 11: e1001739.

15 Kosnett MJ, Succimer (DMSA). In: Brent J, Burkhart K, Dargan P, Hatten B, Megarbane B, Palmer R, eds. Critical Care Toxicology. Springer International Publishing, 2016; 1-5.

16 Dart RC, Hurlbut KM, Maiorino RM et al. Pharmacokinetics of meso-2,3dimercaptosuccinic acid in patients with lead poisoning and in healthy adults. J Pediatr 1994; 125: 309-16.

17 Andersen $\mathrm{O}$. Chemical and biological considerations in the treatment of metal intoxications by chelating agents. Mini Rev Med Chem 2004; 4: 11-21.
18 Alexandre K, Chau F, Guérin F et al. Activity of temocillin in a lethal murine model of infection of intra-abdominal origin due to KPC-producing Escherichia coli. J Antimicrob Chemother 2016; 71: 1899-904.

19 Berleur M, Guérin F, Massias L et al. Activity of fosfomycin alone or combined with temocillin in vitro and in a murine model of peritonitis due to KPC3- or OXA-48-producing Escherichia coli. J Antimicrob Chemother 2018; 73: 3074-80.

20 Lefort A, Chau F, Lepeule R et al. Activity of fosfomycin alone or combined with cefoxitin in vitro and in vivo in a murine model of urinary tract infection due to Escherichia coli harbouring CTX-M-15-type extended-spectrum $\beta$-lactamase. Int J Antimicrob Agents 2014; 43: 366-9.

21 Bleibtreu A, Gros P-A, Laouénan C et al. Fitness, stress resistance, and extraintestinal virulence in Escherichia coli. Infect Immun 2013; 81: 2733-42.

22 Lepeule R, Ruppé E, Le P et al. Cefoxitin as an alternative to carbapenems in a murine model of urinary tract infection due to Escherichia coli harboring CTX-M-15-type extended-spectrum $\beta$-lactamase. Antimicrob Agents Chemother 2012; 56: 1376-81.

23 Signs SA, Tan JS, Salstrom SJ et al. Pharmacokinetics of imipenem in serum and skin window fluid in healthy adults after intramuscular or intravenous administration. Antimicrob Agents Chemother 1992; 36: 1400-3.

24 Aposhian HV. DMSA and DMPS-water soluble antidotes for heavy metal poisoning. Annu Rev Pharmacol Toxicol 1983; 23: 193-215.

25 Couffignal C, Pajot O, Laouénan C et al. Population pharmacokinetics of imipenem in critically ill patients with suspected ventilator-associated pneumonia and evaluation of dosage regimens. Br J Clin Pharmacol 2014; 5: 1022-34.

26 Cavey T, Ropert M, de Tayrac M et al. Mouse genetic background impacts both on iron and non-iron metals parameters and on their relationships. BioMetals 2015; 28: 733-43.

27 Wiskirchen DE, Nordmann P, Crandon JL et al. Efficacy of humanized carbapenem exposures against New Delhi metallo- $\beta$-lactamase (NDM-1)-producing Enterobacteriaceae in a murine infection model. Antimicrob Agents Chemother 2013; 57: 3936-40.

28 Wiskirchen DE, Nordmann P, Crandon JL et al. In vivo efficacy of human simulated regimens of carbapenems and comparator agents against NDM1-producing Enterobacteriaceae. Antimicrob Agents Chemother 2014; 58: 1671-7.

29 Roujansky A, de Lastours V, Guérin F et al. Analysis of paradoxical efficacy of carbapenems against carbapenemase-producing Escherichia coli in a murine model of lethal peritonitis. Antimicrob Agents Chemother 2020; doi: 10.1128/AAC.00853-20.

30 Nordmann P, Cuzon G, Naas T. The real threat of Klebsiella pneumoniae carbapenemase-producing bacteria. Lancet Infect Dis 2009; 9: 228-36.

31 Carrër A, Poirel L, Yilmaz M et al. Spread of OXA-48-encoding plasmid in Turkey and beyond. Antimicrob Agents Chemother 2010; 54: 1369-73.

32 Arikan S, Akkus H, Halifeoglu I et al. Comparison of plasma leptin and zinc levels in elite athletes and sedentary people. Cell Biochem Funct 2008; 26: 655-8.

33 Rotondo CM, Wright GD. Inhibitors of metallo- $\beta$-lactamases. Curr Opin Microbiol 2017; 39: 96-105.

34 Bebrone C. Metallo- $\beta$-lactamases (classification, activity, genetic organization, structure, zinc coordination) and their superfamily. Biochem Pharmacol 2007; 74: 1686-701.

35 Jeon JH, Lee JH, Lee JJ et al. Structural basis for carbapenem-hydrolyzing mechanisms of carbapenemases conferring antibiotic resistance. Int J Mol Sci 2015; 16: 9654-92.

36 Raczynska JE, Shabalin IG et al. A close look onto structural models and primary ligands of metallo- $\beta$-lactamases. Drug Resist Updat 2018; 40: 1-12. 
37 Behari JR, Srivastava S, Gupta S et al. Effect of liposome encapsulated meso-2,3-dimercaptosuccinic acid (DMSA) on biochemical and trace metal alterations in cadmium exposed rats. Bull Environ Contam Toxicol 1991; 47: 827-33.

38 Santos FW, Gonçales CE, Rocha JB et al. 3-Dimercaptopropanol, 2,3dimercaptopropane-1-sulfonic acid and meso-2,3-dimercaptosuccinic acid acute administration differentially change biochemical parameters in mice. Basic Clin Pharmacol Toxicol 2005; 96: 331-4.
39 Asiedu $\mathrm{P}$, Moulton $\mathrm{T}$, Blum $\mathrm{CB}$ et al. Metabolism of meso-2,3dimercaptosuccinic acid in lead-poisoned children and normal adults. Environ Health Perspect 1995; 103.

40 van Eijkeren JC, Olie JD, Bradberry SM et al. Modelling dimercaptosuccinic acid (DMSA) plasma kinetics in humans. Clin Toxicol 2016; 54: 833-9.

41 Farha MA. Brown ED. Drug repurposing for antimicrobial discovery. Nat Microbiol 2019; 4: 565-77. 